\title{
Criminologie
}

\section{La violence dans la communauté militaire}

\section{Deborah Harrison}

Volume 30, numéro 2, automne 1997

Violences familiales

URI : https://id.erudit.org/iderudit/017403ar

DOI : https://doi.org/10.7202/017403ar

Aller au sommaire du numéro

Éditeur(s)

Les Presses de l'Université de Montréal

ISSN

0316-0041 (imprimé)

1492-1367 (numérique)

Découvrir la revue

\section{Citer cet article}

Harrison, D. (1997). La violence dans la communauté militaire. Criminologie, 30(2), 27-45. https://doi.org/10.7202/017403ar

\section{Résumé de l'article}

This paper applies a broad definition of violence to the physically and economically vulnerable situation of Canadian military wives. Its reports some of the results of an institutional ethnography of Canadian military wives ' work which was carried out during the early 1990s, and which made some important discoveries about how the military's priorities and forms of organization structure the way the military treats wives, tries to control wives, and benefits from wives' unpaid work. The military's major priority, combat readiness, necessitates the control the military exercises over its members and the specific mechanism of military control known as combat unit bonding. Combat bonding is in turn characterized by cultural homogeneity and its corollaries of sexism, racism, derogation of, and violence against women. Nevertheless, the military extends a diluted form of combat bonding to military wives, in order to exert control over them and appropriate their unpaid labour, loyalty - and frequently, silence. Combat-related obsession with unit morale also often translates into the cover-up of problems, which is a consideration that adds to the isolation of the many survivors of woman abuse who are members of the military community. The greater public accountability of the Canadian military which may result from the Somalia Inquiry makes this an especially opportune time to study woman abuse and other forms of family violence in the Canadian military community.
Ce document est protégé par la loi sur le droit d'auteur. L’utilisation des services d’Érudit (y compris la reproduction) est assujettie à sa politique d'utilisation que vous pouvez consulter en ligne.

https://apropos.erudit.org/fr/usagers/politique-dutilisation/ 
This paper applies a broad definition of violence to the physically and economically vulnerable situation of Canadian military wives. Its reports some of the results of an institutional ethnography of Canadian military wives' work which was carried out during the early 1990s, and which made some important discoveries about how the military's priorities and forms of organization structure the way the military treats wives, tries to control wives, and benefits from wives' unpaid work. The military's major priority, combat readiness, necessitates the control the military exercises over its members and the specific mechanism of military control known as combat unit bonding. Combat bonding is in turn characterized by cultural homogeneity and its corollaries of sexism, racism, derogation of, and violence against women. Nevertheless, the military extends a diluted form of combat bonding to military wives, in order to exert control over them and appropriate their unpaid labour, loyalty - and frequently, silence. Combat-related obsession with unit morale also often translates into the cover-up of problems, which is a consideration that adds to the isolation of the many survivors of woman abuse who are members of the military community. The greater public accountability of the Canadian military which may result from the Somalia Inquiry makes this an especially opportune time to study woman abuse and other forms of family violence in the Canadian military community.

\section{INTRODUCTION}

Le terme « violence » est un dérivé de « violer », lequel comporte entre autres les trois définitions suivantes, tirées du dictionnaire Oxford : 1) traiter de façon irrévérencieuse ; désacraliser, déshonorer, profaner, ou souiller ; 2) s'approprier par ingérence ; 3) agir sans la considération ou le respect dûment nécessaires. D'autre part, une des définitions du terme « violence » est la suivante : "contrainte indue appliquée à toute forme d'habitus ou de

1. Professeure de sociologie et directrice du Centre de recherche Muriel McQueen Fergusson sur la violence familiale, Université du Nouveau-Brunswick, B.P. 4400, Frédéricton, N.B., E3B 5A3. Adresse électronique : harrison@unb.ca 
processus naturel pour en empêcher le développement ou le libre exercice ". Bien que basé sur un travail empirique, cet article est avant tout une réflexion tentant de relier les définitions précédentes de la violence avec le sexisme culturel de la communauté militaire, ainsi que les conséquences de l'empressement au combat sur les épouses de soldats. Il tend à démontrer que pour bien comprendre la situation de ces femmes, il est indispensable d'utiliser le concept de violence dans un sens assez large pour inclure les abus physiques et psychologiques, ainsi qu'une dépendance économique extrême.

\section{MÉTHODOLOGIE}

Entre 1990 et 1993, Lucie Laliberté ${ }^{2}$ et moi-même avons réalisé une étude nationale sur les épouses de soldats au Canada (Harrison et Laliberté 1993, 1994, 19973). La première étape de cette recherche a consisté à aller rencontrer les communautés militaires en Ontario, au Québec, au NouveauBrunswick, en Nouvelle-Écosse, en Alberta et en Allemagne. Ce sont 112 épouses ou ex-épouses de soldats de tous niveaux et services, anglophones et francophones, qui ont été interviewées. Elles se sont prêtées (de façon confidentielle et anonyme) à des entrevues semi-directives portant sur leur expérience de vie. Grâce à ces entrevues qui nous ont fourni une mine de renseignements très détaillés, nous avons pu établir le schéma de leurs activités quotidiennes en terme de travail; nous avons également étoffé la connaissance sociologique de nouvelles données quant au travail domestique, aux « épouses corporatives", à la violence familiale, et enfin aux femmes âgées, eu égard aux régimes de pension de retraite et à la condition économique.

Ces entrevues nous ont également très bien renseignées sur les opinions des épouses de militaires, en autant qu'un point de vue unifié puisse théoriquement exister. Le " point de vue d'épouse de militaire » implique, à notre sens, la situation d'une femme qui, en épousant un homme, se soumet en même temps au contrôle de l'institution militaire. Nous avons présumé que le bien-être personnel et économique de la femme d'un militaire - sa santé, sa sécurité ainsi que sa capacité à exercer un certain contrôle sur sa

2. Cofondatrice de l'OSOMM (Organization of Spouses of Military Members), Lucie Laliberté est associée au bureau juridique Gahrns \& Laliberté d'Ottawa, lequel se spécialise dans les questions légales touchant la famille et les régimes de pensions de retraite. Elle est également coauteure du livre portant sur les résultats de notre projet de recherche conjoint.

3. Cette recherche a été subventionnée par le Conseil de recherches en sciences humaines du Canada, à travers le Programme stratégique de bourses portant sur la femme et le travail ( $\left.n^{\circ} 882-91-0004\right)$. Cet article est un développement de certaines données de notre recherche. Je tiens à remercier Lucie Laliberté, Carmen Poulin, Walter Schenkel, et les deux évaluateurs anonymes pour leurs commentaires éclairés. 
vie - différaient des besoins de l'institution elle-même. Par la suite, les entrevues ont effectivement démontré que l'indispensable contrôle exercé par l'armée canadienne sur ses membres s'étend inévitablement aux épouses de ceux-ci.

Pour notre recherche, nous avons utilisé la méthodologie sociologique féministe de l'ethnographie institutionnelle telle que développée par Dorothy E. Smith (1986, 1987). Cette méthodologie ethnographique institutionnelle a pour fondement la théorie de l'aliénation de Karl Marx, laquelle implique que tous ceux qui ont été exclus de " l'élaboration d'une idéologie, d'un savoir et d'une culture " se retrouvent piégés dans des systèmes qui définissent sans appel leur mode de vie. Dans un premier temps, l'ethnographie institutionnelle doit donc mettre en lumière l'expérience de vie des personnes dépourvues de pouvoir, pour ensuite resituer ces expériences dans le contexte intellectuel qui leur a donné naissance. Elle doit ensuite tenter de comprendre comment ces expériences reflètent des relations sociales qui, bien qu'invisibles, constituent un important segment de l'ordre social quant aux « relations de domination » (Smith, 1992). Car l'appareil militaire est justement l'une des manifestations de ces relations de domination dans l'ordre social (Mills, 1956).

Pour déterminer de quelle façon l'appareil militaire établit son contrôle sur les épouses, notre recherche ne s'est pas limitée à interviewer 112 épouses de militaires. Le rapport entre le travail des épouses et l'organisation militaire ne peut se comprendre qu'à travers la mise en évidence de la façon dont le travail des épouses profite à l'armée, de la façon dont celle-ci s'assure que le travail des épouses sera fait et, globalement, de la place du travail des épouses dans les buts et structures mêmes des forces armées. À cette fin, après avoir complété les entrevues des épouses, nous avons formulé un nouveau questionnaire tenant compte des données tirées des récits de vie des épouses. Nous avons ensuite soumis ce deuxième questionnaire à 48 militaires ou membres du personnel des forces armées canadiennes. Durant cette seconde étape de notre recherche, nous les avons donc interviewés de la même façon que les épouses, c'est-à-dire verbalement, de manière confidentielle et anonyme, par le biais d'entrevues semi-directives portant sur leur expérience de vie. Nous avons pris leur avis sur les problèmes soulevés par les épouses, ainsi que le sens qu'ils accordent à leur travail au sein de l'armée. Un questionnaire encore plus pointu quant aux orientations visées nous a ensuite permis de poursuivre une démarche ascendante dans la hiérarchie militaire, auprès des superviseurs, des travailleurs sociaux, des prêtres, des médecins, du personnel de soutien aux familles, des administrateurs de programmes, des généraux de haut rang et, pour terminer, auprès du Chef d'état-major de la Défense nationale. Cette troisième phase d'entrevues nous 
a beaucoup renseignées sur les modes d'organisation et les priorités de l'armée. Par ses modes d'organisation et ses priorités, l'armée arrive à contrôler les épouses et à tirer profit de leur travail non rémunéré. Au terme de la recherche, nous avons mis en évidence à quel point les relations de pouvoir au sein des forces armées sont enracinées précisément dans l'expérience de travail quotidien des épouses de militaires. Par conséquent, les aspects de l'institution militaire dont il sera question dans cet article sont sélectifs, puisque ce sont ceux que nous avons identifiés comme ayant le plus d'impact sur les expériences de vie des épouses de militaires canadiens.

\section{L'EMPRESSEMENT AU COMBA'T MILITAIRE}

Considérons maintenant l'institution militaire sous cet aspect et, en premier lieu, parlons de l'obligation d'être « prêts au combat » qui est la tâche essentielle des soldats. Bien que l'empressement au combat fasse aussi référence à l'équipement et au maniement des armes, nous bomerons notre propos aux aspects touchant sa motivation. Pour inculquer et maintenir l'empressement au combat, l'armée doit transformer un humain ordinaire en une sorte d'être qui puisse, en tout temps, être mobilisé à faire la guerre (Grossman, 1995 , p. 13). Elle doit également motiver les épouses de ces personnes (l'armée canadienne compte $89 \%$ d'hommes) à fournir le soutien nécessaire.

Pour atteindre ses fins, la méthode principale de l'armée est celle du contrôle. L'empressement au combat n'est pas inné chez l'homme et bien qu'il soit plus ou moins « pro-militaire », l'environnement civil n'a pas le pouvoir de le transmettre. L'empressement au combat doit donc être inculqué dans un environnement d' " institution totale » où les recrues militaires sont isolées des civils. Pendant les huit semaines de l'entraînement de base (sept semaines pour les officiers), les aspirants échangent leur ancienne identité pour l'uniforme, la coupe de cheveux et les tâches journalières de l'armée. Ils sont humiliés, privés de leurs droits, et dépouillés des réalisations de leur vie antérieure en se faisant répéter continuellement que rien de ce qu'ils ont fait avant le camp d'entraînement n'est important. Le vide ainsi créé dans l'amour-propre de l'aspirant par ce harcelèment est ensuite comblé par la nouvelle identité du «prêt au combat » fournie par l'armée. Peu à peu, les entraîneurs militaires remplacent les agressions verbales par de légers renforcements positifs : à leur admission, les aspirants auront appris à être fiers d'eux-mêmes selon des critères propres à l'armée. Ils auront acquis de

4. Erving Goffman (1961) définit « l'institution totale » comme « un lieu de résidence et de travail où un grand nombre d'individus dans la mêrne situation, coupés du monde extérieur pour une longue période de temps, mènent ensemble un mode de vie reclus sous une administration formelle ". 
nouvelles habiletés et une nouvelle appartenance. Ils auront finalement intégré ce nouveau style de vie qui fait appel aux mécanismes de loyauté enracinés au plus profond d'eux-mêmes.

Après l'entraînement militaire, l'environnement d'institution totale subsiste d'une façon plus atténuée ; les soldats doivent faire fusionner travail et foyer en résidant dans les baraquements de la base militaire. Plusieurs soldats sont déjà mariés et vivent dans les quartiers permanents réservés aux couples, courramment nommés « logements sociaux ». Au cours des années, la plupart des militaires canadiens recevront également des affectations à de nouvelles bases pour éviter qu'ils n'établissent des liens solides avec des civils. D'autre part, les militaires participent intensivement aux rituels et traditions militaires, tels les parades et les rites d'initiation. Ils portent un uniforme distinctif. Ils consultent des prêtres, des médecins, des travailleurs sociaux militaires et non civils. On leur inculque l'idée que les civils sont incapables de comprendre la vie militaire, et on les encourage plus ou moins à s'isoler dans l'armée.

L'encouragement foumi aux soldats visant à ne fratemiser qu'entre eux constitue peut-être le mécanisme préponđérant du contrôle militaire : « le lien de combat ou lien de solidarité au combat ». En cours de combat, la désertion d'un soldat sous le feu peut signifier la mort de tous ceux de son unité. Le lien de solidarité au combat existe pour éviter qu'une telle catastrophe arrive et pour rendre chaque unité de l'armée tellement solidaire qu'en dépit du stress au combat, chaque soldat soit psychologiquement prêt à donner sa vie pour sauver celle d'un autre (Grossman, 1995, p. 148-155).

Ce lien est crucial, dans la culture et l'éthos militaire. Il est construit et maintenu en place par l'isolement des militaires de la vie civile, par les réunions sociales « pour membres seulement », par les absences durant des semaines et des mois (cours, exercices militaires, missions de paix ou combats), par la rigidité de la hiérarchie, son insistance sur l'obéissance aveugle aux supérieurs, son déni des droits civiques tels que la syndicalisation des soldats ou leur implication politique et, de façon plus générale, par une antipathie marquée à l'égard de la vie civile. Le « lien de combat» est encore renforcé par la probabilité que les soldats vont boire ensemble, par les rites d'initiation, par la valorisation des caractéristiques communes aux membres de l'unité (et, inversement, par le dénigrement des autres catégories de personnes) en préparation au combat, où il faudra dénigrer et déshumaniser l'ennemi. Le pluralisme culturel est jugé contraire aux objectifs d'unité dans le combat. Un militaire s'est exprimé ainsi :

Sur la question de l'uniformité, on a été dérangés par l'arrivée des Sikhs... tu fais partie du groupe ou bien tu n'en fais pas partie. C'est 
rien contre toi personnellement, c'est la loi de l'unité. Tu fais partie du groupe ou bien tu n'existes pas... Quand tu vas au camp d'entraînement - même uniforme, même coupe de cheveux, même routine, même style - là tu es intégré. Le mieux, c'est d'être de la même race, d'avoir la même coupe de cheveux, la même religion, les yeux de la même couleur, la même grandeur, le même poids. On aime pas tellement ceux qui sont différents. (Entrevue $\mathbf{n}^{0} 86$ )

Le principe d'exclusivité renforce la cohésion des membres d'une unité de combat par la déshumanisation des membres de groupes sociaux prétendument inférieurs, et ce, en préparation à la déshumanisation de l'ennemi. Cette tendance à la déshumanisation est responsable des rituels de déshumanisation racistes qui ont récemment fait surface (tel le meurtre de Shidane Arone perpétré en Somalie en 1993 par des membres du Régiment canadien aéporté). La culture de l'exclusivité dans l'unité de combat rend acceptables les mauvais traitements infligés à certaines catégories de personnes, parce qu'ils sont perçus comme un renforcement de la solidarité du groupe favorisant l'unité et l'empressement au combat (Grossman, 1995, p. 156-170).

Les femmes sont précisément l'une des catégories de personnes exclues. Comme tout autre organisation sexiste, la communauté militaire prend pour acquise la notion de patriarcat dans la bipolarité homme-femme, ainsi que l'idée de la différence fondamentale entre les hommes et les femmes. Relativement peu de membres de cette communauté remettent en question la dichotomie patriarcale entre l'homme « rude guerrier » et la femme, appui moral et « dépendante ». L'armée se sert de cette construction sociale sur la polarité entre hommes et femmes pour faire de la masculinité le ciment unissant les « vrais » soldats mâles et les distinguer ainsi des non-mâles hommes et femmes ordinaires (Enloe, 1983, 1993). Au cours de l'entrainement, les aspirants masculins sont mis au défi de devenir de " vrais hommes » en démontrant qu'ils ne sont pas des femmes. Les entraîneurs encouragent des comportements masculins stéréotypés de la part des recrues en utilisant un vocabulaire féminin pour les humilier. Les aspirants masculins qui réussissent bien se méritent peu à peu le droit d'être traités en « hommes », et leur loyauté à l'armée se cristallise souvent autour du soulagement d'avoir mérité ce droit. L'exclusion et l'humiliation des femmes sont des aspects importants du lien de solidarité au combat. Les membres d'unités particulièrement « machistes » célèbrent leur masculinité commune en traitant les femmes comme des objets, en regardant des films pornographiques et en faisant des plaisanteries autour des femmes violentées. Quelques membres de ces unités ont déclaré ressentir le même plaisir à violer des femmes qu'à tuer (Grossman, 1995, p. 136-137). 
Le lien de solidarité renforce la cohésion des membres. Plus largement, le lien de solidarité joint les militaires actuels avec ceux du passé, surtout ceux qui sont morts à la guerre. Du point de vue des militaires, le fait de risquer sa vie au combat est le point de divergence majeur entre l'optique de vie des civils et celle des soldats ; c'est aussi un lien puissant entre les soldats actuels et ceux du passé. Selon un militaire :

Nous sommes une vraie société et nous faisons tout entre nous. Par exemple, je n'ai pas besoin d'aller consulter des médecins, dentistes ou avocats civils. Vous comprenez ce que je veux dire ? Tout ce dont j'ai besoin se trouve dans l'armée, je ne pense donc jamais à ce qui est à l'extérieur ; c'est comme notre mode de fonctionnement... les façons d'agir, les parades, le mess. Tout ça existe pour renforcer le lien de solidarité. Plus le travail est dangereux, plus le besoin d'un lien de solidarité est fort. (Entrevue $\mathbf{n}^{\circ} 86$ )

Bien que les autorités militaires n'en conviennent pas ouvertement lorsqu'elles décrivent leur politique officielle, presque toutes les pratiques sont acceptables quand elles visent à renforcer les liens de solidarité entre les membres d'une unité, la volonté commune de vaincre l'ennemi ou même le choix de rester ensemble pour assurer la survie pendant le combat. Partout à travers le monde, les militaires ont résisté à l'intégration des homosexuels et des femmes dans leurs unités, parce qu'ils en craignaient les effets déstabilisateurs sur le plan de l'exclusivité qui est l'essence même du lien de solidarité au combat'.

L'institution militaire repose partiellement sur une dérogation des femmes. Ainsi, elle étend le principe du lien de solidarité au combat aux femmes et aux enfants, afin de mieux contrôler les membres des familles de soldats et pour les conditionner à coopérer et à se coordonner avec le lien des hommes. L'unité qui a bien réussi ce lien de solidarité élargi prend la forme d'une famille patriarcale dans laquelle l'officier commandant est appelé « le patriarche " (the Old Man), sa femme «la mère poule » (The Mother Hen) et les enfants « les jeunes » (the Kids). Dans ces unités comportant un lien fort, les membres se rencontrent souvent, leurs femmes et enfants deviennent amis, et les familles s'entraident au besoin. Peu de civils ont eu l'occasion d'expérimenter la rapidité et l'efficacité avec laquelle les

5. L'armée a toujours résisté aux changements vers une plus grande libéralisation dans la composition du personnel militaire ou dans la structure familiale des militaires. La reconnaissance des femmes combattantes (1989), ou en union libre (1991) et des gais et lesbiennes (1992) n'a pu se produire qu'à travers le succès de contestations juridiques sur les chartes canadiennes des droits de l'homme (pour les combattantes) et des droits et libertés (pour l'union libre et pour les gais et lesbiennes). 
unités militaires organisent des quêtes, distribuent des repas préparés à la maison, envoient des fleurs, visitent les hôpitaux, modifient la destination d'un avion ou nolisent des limousines dans le cas de maladies, d'accidents et autres crises familiales.

Les femmes sont assimilées aux unités de leurs maris à travers la vie sociale, le fait de vivre tous ensemble les moments difficiles, les programmes de soutien aux familles et même la structure hiérarchique de l'armée, qui les encourage à participer à un système social où le rang des épouses les unes par rapport aux autres reflète exactement le rang des hommes dans la structure militaire. Les femmes sont presque automatiquement intégrées dans le lien de solidarité au combat, parce qu'elles ont aussi changé d'affectation à plusieurs reprises, n'ont pas développé de racines civiles significatives et, finalement, ont été encouragées à dépendre de l'armée pour leurs papiers d'identité et souvent pour leur emploi.

Les femmes sont donc intégrées dans la vie sociale de l'armée de telle sorte qu'elles sont en définitive contrôlées par l'armée, et qu'elles contribuent indirectement à la discipline de combat des hommes (Enloe, 1983, p. 40). La philosophie militaire ainsi que trois décennies de recherches en sciences sociales commanditées par l'armée (en particulier aux États-Unis) démontrent que les épouses bien intégrées dans la communauté militaire sont moins susceptibles de se plaindre ou de devenir un fardeau quand leur mari est envoyé en mission. Par ailleurs, elles sont également plus susceptibles de faire leur part pour le bon fonctionnement de l'armée ${ }^{6}$. Citant en exemple les épouses d'un régiment, un général canadien explique ainsi comment fonctionne la mobilisation des conjointes en vue de faciliter l'intégration d'autres femmes et de faire face à l'anxiété quand les maris sont envoyés en mission :

Les épouses des caporaux sont là. Si elles font bien leur travail et parviennent à connaître toutes les autres, tout ira bien. Elles peuvent les connaître par leurs prénoms grâce à ces groupes et associations que toute bonne unité, base. ou camp militaire met sur pied. Lorsque l'une d'entre elles a un problème, elle n'a pas peur d'appeler Marie-je-ne-saisqui, qui est [l'épouse du] sous-officier en chef. Et elle obtiendra toutes sortes de solutions et de conseils. C'est une aide très appréciable. (Entrevue $\mathrm{n}^{0}$ 145)

En premier lieu, les femmes sont intégrées dans l'armée par leur travail. Une part importante du sexisme dans la communauté militaire réside

6. À titre d'exemple, voir Spellman, 1996 ; Montalvo, 1976 ; McKain, 1976 ; Stanton, 1976 ; McCubbin et al., 1978 ; Orthner, 1980 ; Szoc, 1982 ; Kohen, 1984 ; Segal, 1986 ; Orthner et al., 1986 ; Pittman et al., 1988 ; Bowen et al., 1989 ; Orthner et al., 1990 ; Bell et al., 1991, et Campbell et al., 1991. 
dans son appropriation de l'idéologie patriarcale pour laquelle la division du travail selon les sexes est naturelle. Cette division sexuée du travail est vraiment la pierre angulaire de la communauté militaire canadienne. Dès lors, on prend pour acquis que chaque épouse de soldat a) assumera l'entièreté du travail domestique et du soin des enfants pendant les nombreux mois de l'année où son mari sera en mission; b) contrebalancera les effets déstabilisateurs au plan familial de l'absence du mari en ne recherchant pas de travail rénuméré ou en n'y accordant pas une importance primordiale ; c) abandonnera son travail chaque fois que son mari recevra une nouvelle affectation ; d) accomplira tout le travail non rémunéré associé à la nouvelle affectation, tels les emballages et déballages du déménagement, la recherche du logement, de l'école et de groupes d'amis pour ses enfants ; e) comblera le vide créé par sa faible implication sur le marché du travail en faisant des travaux peu rémunérés - et même du bénévolat - à la base militaire, et f) (pour les femmes d'officiers supérieurs) consacrera un temps considérable à entraîner et superviser les autres épouses ainsi qu'à représenter l'armée dans les œuvres de bienfaisance civiles. Le même général résume ainsi les contributions sous-jacentes à l'effort militaire que l' armée canadienne attend des épouses :

Oh ! les épouses sont vraiment dans l'armée ! Si un militaire marie une femme qui ne comprend pas qu'elle fait partie de l'armée dans le sens qu'elle devra déménager et faire une foule de choses qu'elle ne ferait pas autrement, ou bien le mariage ne durera pas, ou bien elle devra changer de point de vue. Ici, on se marie avec l'institution ! (Entrevue $n^{\circ} 145$ )

La plupart des épouses de militaires sont incapables de « faire carrière », dans le sens de la classe moyenne, ou même d'occuper un emploi assez longtemps pour que les contributions à leur régime de pension de retraite soient versées. Leur contribution au marché du travail est beaucoup plus faible que celle des épouses civiles'. Toutefois, l'institution militaire témoigne beaucoup plus d'estime aux épouses qui œuvrent bénévolement dans les programmes d'aide aux familles qu'à celles qui assument un travail bien rémunéré à l'extérieur de la base militaire, et qui s'insurgent lorsque le mari est transféré. L'armée approuve de tout cœur les épouses qui dédaignent les offres d'emploi bien rémunéré à l'extérieur afin de se consacrer à la communauté militaire. Voici ce qu'en pense un officier canadien :

7. $53 \%$ des épouses de militaires canadiens n'occupent aucun emploi à l'extérieur de la maison, ce qui n'est le cas que pour $23 \%$ des canadiennes en général (Organization of Spouses of Military Members, 1992). 
Je dis souvent qu'il y a plusieurs sortes d'épouses militaires. D'abord, il y a celles qui ne peuvent et ne pourront jamais s'y faire. Ensuite, il y a celles qui n'y arrivaient pas et qui ont trouvé un accommodement. D'habitude celles-là sont parties de la base, ont commencé une nouvelle carrière à l'extérieur des quartiers permanents réservés aux couples et leur mode de fonctionnement n' apporte aucune solution aux problèmes. Finalement, il y a celles qui ont appris à s'y faire et qui contribuent à régler les problèmes. (Entrevue $\mathrm{n}^{\circ}{ }^{96}$ )

Selon le point de vue des militaires interviewés, un grand nombre d'épouses « trouvent des solutions aux problèmes ». À un coût énorme pour elles-mêmes, ces femmes deviennent et restent loyales à l'armée ; elles entrent dans le lien de solidarité avec la communauté militaire et se plient aux attentes de l'armée en accomplissant des tâches mal rémunérées.

\section{AUTRES ASPECTS DU CONTRÔLE MILITAIRE}

Le lien de solidarité au combat n'est pas la seule forme de contrôle exercée par l'armée sur ses membres ayant une implication sur leurs épouses. $\mathrm{La}$ « nature à somme zéro du combat » et le sens de la « morale au combat » sont deux autres modes de contrôle militaire.

Le combat a pour fondement un modèle de contrôle à somme zéro selon lequel la réussite implique une prise de contrôle sur les autres en termes de vie ou de mort. L'efficacité d'une unité de combat est souvent jugée à la capacité de son commandant à « prendre le contrôle ». Aux yeux de l'armée, un ordre donné par une personne en autorité est souvent l'élément indispensable pour sauver une unité de combat en danger de mort. Selon un membre de la marine canadienne :

Aux îles Falkland, un bateau a été touché - éventré. Le choc a été terrible, bien que personne n' ait été tué. Écoutez, on parle de 600 a 900 livres d'explosifs qui ont atteint le bateau, qui l'ont littéralement soulevé de l'eau. Ce qu'ils ont fait immédiatement - on l'a su par la suite - ça a été d'envoyer par avion un officier en chef — un ingénieur parce que là-bas, tout le monde était sonné. Aussitôt qu'il est arrivé et qu'il a commencé à donner des ordres, tout le monde a repris ses sens. C'est à ça que servent les manœuvres militaires, vous comprenez ? «Quelqu'un est en charge : je n'ai rien à craindre ». (Entrevue $n^{\circ} 86$ )

La plupart des femmes qui subissent des abus de la part de leur conjoint les décrivent comme obsédés par le contrôle. Bien que plusieurs abuseurs obsédés de contrôle ne soient pas des militaires, il est évident que l'armée est l'une des institutions où les comportements de contrôle sont les plus valorisés. C'est également un lieu où un militaire perçu comme incapable 
de «contrôler sa femme» est menacé de perdre la face. L'obsession de contrôle vient donc renforcer le caractère misogyne du lien de solidarité au combat, tout en contribuant au nombre relativement élevé de femmes abusées dans la communauté militaire (Neidig, 1985 ; MacLeod, 1987 ; Comité canadien sur la violence faite aux femmes, 1993 ; Thompson, 1994 ; Cronin, 1995).

De plus, dans les unités qui opèrent sur le terrain à proximité d'un conflit armé, il est jugé nécessaire que tous les soldats partagent une attitude détachée face à la violence, afin que chacun soit convaincu de pouvoir se fier entièrement aux autres pendant la bataille, au point de ne pas fléchir lui-même (Starr, 1982). Des actes de violence collective en sont parfois la terrible conséquence. Au Canada, nous avons connu l'exemple du meurtre de Shidane Arone, et aux États-Unis, le viol collectif de soldates, pendant la Convention militaire américaine Tailhook, en 1991. La violence familiale constitue un autre exutoire situationnel fréquent. Un numéro récent du New York Times mentionne qu' aux États-Unis, une femme ou un enfant est tué chaque semaine par un proche parent, membre des forces armées. On y cite les données d'un rapport américain selon lequel une famille militaire sur trois a connu des épisodes de violence aux États-Unis ${ }^{8}$. Une étude américaine récente a comparé 30 couples militaires à 30 couples civils : $23 \%$ des épouses de soldats ont déclaré avoir été battues, en comparaison de seulement $3 \%$ des épouses civiles du groupe de contrôle (Griffin et Morgan 1988). D'autres études américaines ont indiqué que le nombre de femmes battues est particulièrement élevé chez les militaires de carrière (Brown et al., 1981 ; Starr, 1982 ; Neidig, 1985 ; Baron et Straus, 1987 ; Shupe et al., 1987 ; Maloney, 1988 ; Solomon, 1988 ; Cook, 1990 ; Gondolf et al., 1991). Pendant nos entrevues, nous avons entendu tant d'histoires choquantes de la part d'épouses ayant survécu à des mariages violents que nous avons tout lieu de croire que la situation est identique au Canada.

La considération morale est élevée dans l'armée ; elle est perçue comme devant s'élever automatiquement avec le grade de celui qui donne les ordres et qui doit gagner la confiance de ses subalternes. En conséquence, la dissimulation des erreurs d'un supérieur est souvent considérée comme justifiable pour des motifs opérationnels. La culture militaire aborde en anecdotes sur ce thème : dissimulation des fautes du partenaire (enseignée aux recrues dès l'entraînement de base), de celles d'un supérieur et même de celles de l'armée en tant que telle, en taisant les mauvais côtés de l'armée pour ne présenter

8. Voir « Military Struggling To Stem an Increase in Family Violence", New York Times, 23 mai 1994. 
au monde extérieur qu'une image sans défaut. Comme la plupart des autres aspects de la culture militaire, la nécessité d'une apparente perfection est liée au besoin de contrôle de l'armée. Dans son livre Military Brats (1991), Mary Edwards Wertsch observe ce qui suit :

L'état d'esprit militaire adéquat est celui dans lequel on est prêt à prendre le contrôle de toute situation, quelles qu'en soient les données. De plus, l'état d'esprit militaire adéquat est de toujours avoir l'air, et d'agir comme si on était prêt pour cette mission-ci. Tout comme si, avec leur apparence soignée et leurs comportements automatisés, les soldats disaient : « En voyant comment nous nous contrôlons nous-mêmes, vous comprendrez comment nous pouvons contrôler l'ennemi » (p. 34).

Selon certains militaires interviewés, quelques-uns des aspects primordiaux de la guerre sont d'ordre psychologique. En ce sens l'apparence de perfection est cruciale. L'uniforme impeccable, les souliers bien cirés, la coordination parfaite de chaque individu de la brigade marchant au pas cadencé dans une formation de parade, tout cela contribue subrepticement à l'affrontement et à l'intimidation de l'ennemi. L'apparence de perfection dans la coordination de 5000 hommes levant leurs fusils à l'unisson est conçue pour détruire l'assurance de l'adversaire. Dans la discipline militaire, tout ce qui devrait être passé sous silence pour ne pas nuire à l'image sans faille de l'armée sera, la plupart du temps, caché. Tout comme pour le lien de solidarité au combat, on croit que cette perfection apparente est une condition indispensable à la survie des soldats. Un marin s'est servi de l'exemple de la crise d'Oka au Canada pour expliquer comment une apparence sans faille détruit l'assurance de l'ennemi :

Oka, vous vous souvenez ? On s'est montrés avec 5000 hommes - une brigade. Ce que ça a donné ? On a détruit leur détermination, leur désir de combattre... C'est ça que ça a donné. Le résultat, c'est la destruction de la confiance en soi, c'est ça qui est en cause. On démontre une force imbattabie : "îls n'ont aucune chance de gagner. » On a détruit leur détermination. (Entrevue $\mathrm{n}^{\circ} 86$ )

La dissimulation en vue d'une perfection apparente débute au camp d'entraînement, où tout le monde est puni pour la faute d'une seule recrue. Ainsi, il devient vite évident à chacun des membres de l'escadron qu'il est dans son intérêt personnel de dissimuler les erreurs de ses pairs. Par la suite, les recrues apprennent aussi à taire les erreurs de leurs supérieurs. C'est l'unique solution possible, étant donné qu'en cas de conflit, la seule version des faits retenue sera celle du supérieur. Au cours de nos entrevues, nous avons eu l'exemple d'un simple soldat qui a dû taire l'agression sexuelle commise par un officier superieur sur son propre enfant. Dans une autre occasion, un 
capitaine de l'air a reçu l'ordre de prendre tous les moyens pour obliger ses inférieurs à assister à une danse très impopulaire, simplement pour que le colonel qui l'avait organisée ne perde pas la face. Autre exemple : un marin blessé à la tête par un supérieur lors d'une bagarre a été sommé de se taire sous peine d'être renvoyé. Selon la philosophie de l'armée canadienne, ceux qui donnent les ordres doivent paraître infaillibles pour être obéis. Cette convention sociale autour de l'infaillibilité est considérée comme essentielle au maintien du moral des troupes et à la prévention de mutineries. Voici le commentaire d'un officier supérieur :

Il est essentiel d'avoir toute confiance dans les supérieurs car un jour, ils pourraient vous demander d'accomplir quelque chose de très dangereux. Nous nous entraînons pour la guerre et non pour la paix. Finalement, la justification c'est qu'un jour il faudra faire appel à cette confiance absolue de la part de nos hommes. (Entrevue $n^{\circ} 26$ )

L'apparence d'infaillibilité repose en majeure partie sur le secret. Cette considération aide à comprendre la tendance de l'armée à serrer les rangs lorsque des abus ont été commis. De même, chaque militaire apprend que la divulgation de ses problèmes personnels pourrait ternir le moral de son unité à l'interne et/ou la réputation militaire à l'extérieur. Malgré la position officielle de l'armée selon laquelle « il faut en parler », les militaires sont plutôt dissuadés de venir exposer leurs problèmes personnels, à moins que ceux-ci ne semblent porter préjudice à leur performance au travail. En conséquence, le soldat alcoolique ou violent a tout lieu de faire taire sa femme.

L'épouse d'un soldat alcoolique ou violent a ses propres raisons de se taire : la honte, la peur de sévices physiques encore pires ou même la perception (souvent justifiée) que personne dans la communauté militaire ne veut l'entendre. Une telle épouse a souvent aussi été mutée plusieurs fois avec son mari, elle a perdu tous les liens avec son ancien milieu civil et elle en est venue à se dire que la communauté militaire ainsi que le chèque de paie de son mari représentent sa seule sécurité. Cette femme peut vraisemblablement craindre pour sa vie. Par ailleurs, l'épouse qui, surmontant ses craintes, est assez courageuse pour rechercher de l'aide à la base militaire, souvent ne sera pas crue par l'aumonier ou le travailleur social. Ces membres du personnel militaire ont des motifs liés à leur propre carrière de maintenir le lien de solidarité et de soutenir les soldats incriminés. Plusieurs d'entre eux n'ont d'ailleurs aucune connaissance des dynamiques liées à l'abus des femmes. Si l'épouse fait appel à un professionnel civil de l'extérieur, souvent, celui-ci la dirigera vers l'armée. Il ne fait aucun doute que l'un des côts les plus élevés du lien de solidarité au combat, en termes de qualité de vie, est celui de l'isolement psychologique et de la vulnérabilité physique des épouses de militaires ayant survécu aux abus. 


\section{VULNÉRABILITÉ ÉCONOMIQUE}

Toutes les épouses ne sont pas victimes de violence. Mais presque toutes subissent les vicissitudes du mode de vie militaire, en termes de vulnérabilité économique. Les efforts de l'armée, souvent couronnés de succès, pour inclure les épouses dans le lien de solidarité (décrit ci-dessus), induisent les femmes dans un faux sentiment de sécurité. Elles croient que l'armée va toujours prendre soin d'elles et qu'elles appartiendront toujours à la famille militaire. Au cours de son mariage, la femme est encouragée à se laisser prendre en charge et à s'installer dans le confort prévisible offert par la communauté militaire : associations, logement, vie sociale interne et partage d'un univers commun. Elle croit que les risques associés au fait de suivre son mari seront minimes, puisque à chaque étape, l'armée fournit la continuité, l'intimité et un cercle de nouveaux amis présélectionnés. Elle a le sentiment de pouvoir « suivre le courant», puisque, libre de faire son travail, son mari obtiendra des promotions dont bénéficiera la famille. Elle-même sera perçue comme un « actif » dans sa carrière, et l'armée fera le reste. Elle est bercée par l'illusion qu'elle n'accomplit pas un travail ou un sacrifice mais simplement sa part, dans une communauté qui en fait de même pour elle.

Dans le cas où son mariage prend fin, l'épouse militaire canadienne éprouve un véritable choc. En comparaison avec les épouses civiles dans la même situation, elle subit une chute phénoménale de son statut économique, particulièrement parce qu'il est peu probable qu'elle ait conservé sa compétence au travail ou acquis son propre capital ou régime de pension de retraite au cours de son mariage. En dépit de tous les sacrifices consentis, l'épouse séparée est traitée comme n'ayant jamais travaillé pour l'armée mais plutôt comme ayant simplement rendu service à un quelconque individu (Smith, 1985 ; Burke et Spector, 1991). Au moment de la séparation, le lien de solidarité refait surface pour se resserrer autour du militaire en même temps qu'il ferme la porte à son épouse. Un sous-officier de la marine résume ainsi l'attitude de l'armée envers la femme séparée ou divorcée :

On va tenter quelque chose, si on est au courant. On va se fendre en quatre pour vous, pour votre épouse. La femme d'un type arrive, elle a un problème, son mari doit partir pour le Moyen-Orient... bon ! On va aller pelleter ses escaliers. On va faire plein de trucs, du moins dans ma base militaire. Mais si elle est pour divorcer, se séparer ou partir, alors elle ne fait plus partie du groupe. Elle a quitté la famille. Bing, bang ! C'est comme ça que ça marche. Nous, on fonctionne de l'intérieur. Je vous le dis, on fonctionne de l'intérieur. (Entrevue $n^{\circ} 86$ )

Toutes les forces armées ont été lentes à reconnaitre que le travail de soutien non rémunéré assumé par les épouses de militaires était vraiment du 
travail, que les femmes divorcées ou séparées devaient recevoir une compensation pour les tâches accomplies - ainsi que pour les offres d'emploi perdues au civil - et que le régime de pension de retraite est une forme de rémunération différée aux deux conjoints plutôt que la propriété exclusive du mari ${ }^{9}$.

Les forces armées ont également été lentes à reconnaître que l'épouse dont l'ancrage social avait été pendant des années la communauté militaire, était, au moment de sa séparation, une personne laissée cruellement à l'abandon. Une ex-épouse de militaire canadien explique comment elle s'est sentie au moment de la rupture de son mariage :

Je n'avais pas d'identité. Je n'étais pas moi-même. J'étais le quelque chose de quelqu'un d'autre. J'étais Mme sous-officier en chef, vous comprenez ? Et je ne le réalisais même pas. Je pensais que c'était comme ça que ça devait être. Je pensais vraiment que c'était comme ça que ça devait être. Et puis, tout d'un coup, on n'a plus eu besoin de moi. On m'a enveloppée dans un journal et mise à la poubelle comme un rebut. (Entrevue $n^{\circ} 14$ )

\section{CONCLUSION}

Selon les militaires que nous avons interviewés, l'empressement au combat est la justification ultime de la plupart des règlements imposés et sanctionnés par l'armée. L'empressement au combat exige que l'armée contrôle ses membres et mette en place les mesures nécessaires pour s'assurer leur obéissance et leur loyauté. Ce sont les expériences de vie relatées par les épouses qui nous ont permis de cibler les informations à recueillir auprès des militaires.

Le contrôle de l'armée sur ses membres est estimé reposer sur une combinaison d'éléments tels la hiérarchie, la loyauté envers l'institution militaire et, finalement, le lien profond de l'individu avec son unité, particulièrement dans les unités de combat. On croit que l'homogénéité culturelle est un prérequis important à la force du lien de solidarité militaire ; le respect de ce prérequis a donc souvent pour corollaire le racisme et le sexisme. Les célébrations machistes dans les unités de combat et, conséquemment, le

9. Pour de plus amples informations sur les droits de l'épouse séparée ou divorcée quant au régime de pension de retraite d'un militaire, voir Harrison et Laliberté, 1994, chapitre 7. Les règlements concemant l'Acte de division des bénéfices de la pension militaire ont été rendus publics pendant que le livre était sous presse. Subséquemment, ces règlements ont été modifiés afin de favoriser davantage les épouses séparées et divorcées, résultat du lobbying de l'Organisation des épouses de militaires. 
dénigrement des femmes sont des caractéristiques importantes du sexisme dans la communauté militaire.

L'armée privilégie une approche de contrôle sur ses membres; le contrôle est l'essence même de l'action militaire. Le modèle de contrôle à somme zéro prôné par l'armée fournit des renforcements positifs au soldat qui réussit à contrôler les situations difficiles ; « l'épouse récalcitrante » en fait partie. Affirmer que les comportements violents sont valorisés dans les unités de combat serait inadéquat : dans ces unités, la violence contre les femmes est aussi spécifiquement tolérée et entretenue.

En dépit de ses tendances misogynes, l'armée s'attend, de la part des épouses, à des comportements de loyauté et d'adhésion à l'institution. Conséquemment, plusieurs épouses sont fidèles à l'armée, plusieurs profitent de la camaraderie de la communauté militaire, avec comme résultat une difficulté accrue pour les femmes abusées à obtenir de l'aide à l'intérieur de la communauté. Dans l'armée, l'obsession de ne pas diminuer le moral des troupes se traduit souvent par une dissimulation des problèmes. Pour les épouses ayant survécu à la violence, cette attitude constitue une difficulté supplémentaire à obtenir une écoute favorable de la part du personnel de soutien des forces militaires.

Par « violence", nous faisons habituellement référence aux abus physiques et psychologiques. Toutefois, notre conception de la violence militaire devrait être plus large, comme nous le rappelle l'inclusion de « l'appropriation » et de « la contrainte » à la définition de la violence donnée au début de cet article. La vulnérabilité économique des épouses militaires et la triste condition économique de la plupart des épouses séparées et divorcées sont également des formes de violence, dans le sens d'appropriation ou de contrainte « appliquées à un processus naturel » pour « empêcher le développement ou le libre exercice ". Le mode de vie militaire empêche la plupart des épouses de gagner leur vie d'une manière appropriée à leurs capacités. Plusieurs femmes mariées connaissent la même vulnérabilité économique dans le civil. Cependant, la combinaison de la mobilité géographique dans l'armée et la forte proportion de travaux non rémunérés exigés des épouses militaires rendent leur situation économique extrêmement précaire.

À moins que l'armée ne soit forcée de prendre ses responsabilités au sujet de la violence contre les épouses, le secret institutionnalisé va continuer de prévaloir, et les femmes maltraitées continueront de souffrir dans un isolement intolérable. L'intérêt soulevé par la présente enquête sur les événements de Somalie démontre que le public trouve inacceptable le point de vue selon lequel l'armée seule est concernée par ses erreurs. Les événements de Somalie auront potentiellement l'effet de soumettre les agissements de 
l'armée canadienne à un examen plus rigoureux de la part du public. Ce contexte foumit donc une occasion unique pour étudier davantage la violence et les autres formes d'abus contre les femmes, tant dans la communauté militaire que civile. Il est urgent d'en tenir compte.

\section{BIBLIOGRAPHIE}

BARON, L. et STRAUS, M. A. (1987), «Four Theories of Rape : A Macrosociological Analysis ", Social Problems, $n^{\circ} 34$, p. 467-489.

BELL, D. B., SCARVILLE, J. et QUIGLEY, B. (1991), The Army Family Research Program : Origin. Purpose and Accomplishments, Projet de l'armée numéro 2Q263731A792, Alexandria, VA : United States Army Research Institute for the Behavioral and Social Sciences.

BOWEN, G. et NEENAN, P. (1989), « Organizational Attitude Towards Families and Satisfaction With the Military as a Way of Life : Perceptions of Civilian Spouses of U.S. Army Members ", Family Perspectives, vol. 23, na 1, p. 2-13.

BROWN, M. M., AGUIRRE, B. E. et JORGENSEN, C. (1981), " Abusers of Clients of Wornen's Shelter : Their Socialization and Resources ", Joumal of Sociology and Social Welfare, vol. 8, $\mathbf{n}^{\circ} 3$ (septembre), p. 462-470.

BURKE, M. A. et SPECTOR, A. (1991), «Une catégorie de personnes défavorisées : les femmes âgées de 55 à 64 ans qui vivent seules ", Tendances sociales canadiennes, $\mathrm{n}^{\circ} 23$ (hiver), p. 14-17.

CAMPBELL, C. H., CAMPBELL, R.C., RAMSBERGER, P., SCHULTZ, S., STAWARSKI, C. et STYLES, M. (1991), A model of Family Factors and Individual and Unit Readyness : Literature Review, Note de recherche $\mathbf{n}^{\circ}$ 91-30, Alexandria, VA : United States Amy Reseach Institute for the Behavioral and Social Sciences.

CANADA-MINISTĖRE DELA DÉFENSE NATIONALE (1994), Livre blanc sur la défense, Ottawa, Approvisionnements et Services Canada.

COMITÉ CANADIEN SUR LA VIOLENCE FAITE AUX FEMMES (1993), Nouvel horizon : éliminer la violence - atteindre l'égalité, Ottawa, Approvisionnements et Services Canada.

COOK, K. J. (1990), «Cultural Spillover Theory and Violence in the Family : the Case of the Military », communication présentée aux rencontres de la Société américaine de criminologie.

CRONIN, C. (1995), “ Adolescent Reports of Parental Spousal Violence in Military and Civilian Families ", Journal of Interpersonal Violence, vol, 10, $\mathrm{n}^{\circ} 1$ (mars), p. 117 122.

ENLOE, C. (1983), Does Khaki Become You? The Militarization of Women's Lives, Boston, South End Press.

ENLOE, C. (1993), The Morning After : Sexual Politics at the End of the Cold War, Berkeley, University of California Press.

GOFFMAN, E. (1961), Asylums : Essays on the Social Situation of Mental Patients and Other Inmates, New York, Doubleday.

GONDOLF, E. W. et FOSTER, R. A. (1991), «Wife Assault Among VA Alcohol Rehabilitation Patients ", Hospital and Community Pychiatry, vol. $42, \mathrm{n}^{\circ} 1$ (janvier), p. 74-79. 
GRIFFIN, W. A. et MORGAN, A. R. (1988), «Conflict in Maritally Distressed Military Couples ", American Journal of Family Therapy, vol. 16, $\mathrm{n}^{\circ} 1, \mathrm{p} .14-22$.

GROSSMAN, Lt-Col D. (1995), On Killing : The Psychological Cost of Learning to Kill in War and Society, Boston, Little, Brown and Company.

HARRISON, D. et LALIBERTÉ, L. (1993), « How Combat Ideology Structures Military Wives' Dornestic Labour ", Studies in Political Economy, $n^{\circ} 42$ (Automne), p. 45-80.

HARRISON D. et LALIBERTÉ, L. (1994), No Life Like It: Military Wives in Canada, Toronto, Jarnes Lorimer and Company.

HARRISON D. et LALIBERTÉ, L. (1997), " Gender, the Military, and Military Family Support ", dans Laurie Weinstein and Christine White (éd.), Wives and Warriors, Westport, Ct., Greenwood (sous presse).

KOHEN, J. A. (1984), « The Military Career is a Family Affair ", Journal of Family lssues vol. $5, n^{\circ} 3$ (septembre), p. 401-418.

MACLEOD, L. (1987), Pour de vraies amours... prévenir la violence conjugale, Ottawa, Conseil consultatif canadien sur la situation de la femme.

MALONEY, L. J. (1988), « Post Traumatic Stresses on Women Partners of Vietnam Veterans ", Smith College Studies in Social Work, vol. 58, $n^{\circ} 2$ (mars), p. 122-143.

MCCUBBIN, H. I., MARSDEN, M.A., DURNING, K. P. et HUNTER, E. J. (1978), « Family Policy in the Armed Forces: An Assessment ", Air University Review, (septembreoctobre), p. 46-57.

MCKAIN, J. L. (1976), « Alienation : A Function of Geographical Mobility Among Families ", dans H. I. McCubbin et al. (ed.), Families in the Military System, Beverly Hills, Sage, p. 69-91.

MILLS, C. W. (1956), The Power Elite, New York, Oxford University Press.

MONTALVO, F. F. (1976), « Family Separation in the Army : A Study of the Problems Encountered and the Caretaking Resources Used by Career Amny Farnilies ", dans H. I. McCubbin et al. (éd.), Families in the Military System, Bevery Hills, Sage, p. 147173.

NEIDIG, P. H. (1985), « Domestic Violence in the Military, Part II : the Impact of High Levels of Work-Related Stress on Family Functionning ", Military Family, juillet-août, p. 3-5.

NEW-YORK TIMES (1994), « Military Struggling to Stem on Increase in Family Violence », 23 mai.

ORGANIZATION OF SPOUSES OF MILITARY MEMBERS (1992), Brief to the Senate Finance Committee on Bill C-55, Respecting the Pension Benefits Division Act, Ottawa, septembre.

ORTHNER, D. K. (1980), Families in Blue : a Study of Married and Single Parent Families in the Air Force, Washington, Department of the Air Force.

ORTHNER, D. K. et PITTMAN, J. F. (1986), « Family Contribution to Work Commitment », Joumal of Marriage and the Family, $\mathrm{n}^{\circ} 48$ (août), p. 573-581.

ORTHNER, D. K., POLLOCK, D. L., EARLY-ADAMS, P., DEVALL, E., GIDDINGS, $M$. et MORLEY, R. (1990), Building Strong Army Communities, Note de recherche n 90 110, Alexandria, VA, United State Arny Research Institute for the Behavioral and Social Sciences.

PITTMAN, J. F. et ORTHNER, D.K. (1988), « Predictors of Spousal Support for the Work Commitments of Husbands », Joumal of Marriage and the Family, $\mathrm{n}^{\circ} 50$ (mai), p. 335348. 
SEGAL, M. W. (1986), « The Military and the Family as Greedy Institutions ", Armed Forces and Society, vol. 13, $\mathrm{n}^{\circ} 1$ (automne), p. 9-38.

SHUPE, A., WILLIAM, A. S. et HAZIEWOOD, L. R. (1987), Violent Men, Violent Couples : The Dynamics of Domestic Violence, Lexington, MA, Lexington Books.

SMITH, D. E. (1985), "Women, Class and Family », dans Varda Burstyn et al., (éd.), Women, Class, Family and the States, Toronto, Garamond, p. 1-44.

SMITH, D. E. (1986), «Institutional Ethnography : A Feminist Method », Resources for Feminist Research, vol. 15, $\mathrm{n}^{\circ}$ 1, p. 6-13.

SMITH, D. E. (1987), The Everyday World as Problematic : A Feminist Sociology, Toronto, University of Toronto Press.

SMITH, D. E. (1992), "Ferninist Reflections on Political Economy ", dans M. Patricia Connelly et al., (éd.), Feminism in Action: Studies in Political Economy, Toronto : Canadian Scholars' Press, p. 1-21.

SOLOMON, Z. (1988), « The Effects of Combat-Related Posttraumatic Stress Disorder on the Family 》, Psychiatry, no 51 (août), p. 323-329.

SPELLMAN, S. (1976), «Utilization of Problem-Solving Resources Among Military Families ", dans H. I. McCubbin et al., (éd.), Families in the Military System, Beverly Hills, Sage, p. 174-206.

STANTON, D. H. (1976), « The Military Family : Its Future in the All-Volunteer Context », dans Nancy Goldman et al., (éd.), The Social Psychology of Military Service, Beverly Hills, Sage, p. 135-149.

STARR, P. D. (1982), « Military Socialization in the University : The Role of Subcultures in Navy-Marine ROTC », Human Organization, vol. 41, $n^{\circ} 1$ (printemps), p. 64-69.

SZOC, R. (1982), Family Factors Critical to Retention, San Diego, Naval Personnel Research and Development Center.

THOMPSON, M. (1994), « Armed Forces : The Living Room War », Time, vol. 143, n² 21 (23 mai), p. 48-51.

TORONTO STAR (1992), «Woman Pilot Recalls Terror of Running Naval Sex Gantlet », 25 juin.

WERTSCH, M. E. (1991), Military Brats : Legacies of Childhood Inside the Fortress, New York, Random House. 